\title{
Efficacy and Safety of a Single-Pill Combination of Vildagliptin and Metformin in Japanese Patients with Type 2 Diabetes Mellitus: A Randomized, Double-Blind, Placebo-Controlled Trial
}

\author{
Masato Odawara • Mika Yoshiki • Misako Sano • Izumi Hamada • \\ Valentina Lukashevich • Wolfgang Kothny
}

To view enhanced content go to www.diabetestherapy-open.com Received: December 24, 2014 / Published online: February 18, 2015

(C) The Author(s) 2015. This article is published with open access at Springerlink.com

\section{ABSTRACT}

Introduction: The use of dipeptidyl peptidase-4 inhibitors in combination with metformin is increasing in Japanese patients with type 2 diabetes mellitus (T2DM), but no single-pill combination (SPC) is currently available in Japan. The objective of this study was to assess the efficacy and safety of vildagliptin/ metformin SPC in Japanese patients with T2DM inadequately controlled with vildagliptin monotherapy.

Trial registration: ClinicalTrials.gov, \#NCT01811485.

Electronic supplementary material The online version of this article (doi:10.1007/s13300-015-0099-x) contains supplementary material, which is available to authorized users.

M. Odawara $(\bowtie)$

The Department of Diabetes, Endocrinology,

Metabolism and Rheumatology,

Tokyo Medical University, Tokyo, Japan

e-mail: odawara@tokyo-med.ac.jp

M. Yoshiki · M. Sano · I. Hamada

Novartis Pharma K.K., Tokyo, Japan

V. Lukashevich

Novartis Pharmaceuticals Corporation,

East Hanover, NJ, USA

W. Kothny

Novartis Pharma AG, Basel, Switzerland
Methods: This was a 14-week, randomized, double-blind, parallel-group, placebocontrolled trial. 171 patients with T2DM inadequately controlled [HbA1c (glycosylated hemoglobin) 7.0-10.0\%] with vildagliptin $50 \mathrm{mg}$ twice daily (bid) were randomized (2:1) to receive either a vildagliptin/metformin SPC $(n=115)$ or matching vildagliptin/placebo SPC $(n=56)$.

Results: Baseline demographics and background characteristics were generally comparable between the treatment groups. The change in HbA1c [mean \pm standard error (SE)] was $-0.8 \pm 0.1 \%$ in the vildagliptin/ metformin SPC (baseline HbA1c, $7.9 \pm 0.1 \%$ ) group and $0.1 \pm 0.1 \%$ in the vildagliptin/placebo SPC (baseline HbA1c, $8.0 \pm 0.1 \%)$ group, with a between-treatment difference of $-1.0 \pm 0.1 \%(P<0.001)$ in favor of the vildagliptin/metformin SPC group. The proportion of patients achieving target HbA1c $<7.0 \%$ was significantly higher with vildagliptin/metformin SPC compared with vildagliptin/placebo SPC $(45.8 \%$ vs. $13.5 \%$, $P<0.001)$. The overall incidences of adverse events (AEs) were $43.5 \%$ in the vildagliptin/ metformin SPC and $67.9 \%$ in the 
vildagliptin/placebo SPC group. The incidences of serious AEs were low in both the treatment groups $(0.9 \%$ vs. $3.6 \%$, respectively). Body weight remained constant throughout the study in both the treatment groups. There were no deaths or hypoglycemic events during the study.

Conclusions: Switching Japanese patients with T2DM requiring treatment intensification, from vildagliptin monotherapy to a vildagliptin/ metformin SPC (50/250 or $50 / 500 \mathrm{mg})$ was efficacious and safe, eliciting significant reduction in HbA1c without increased risk of hypoglycemia and weight gain.

Keywords: Dipeptidyl peptidase-4 inhibitor; Metformin; Single-pill combination; Type 2 diabetes mellitus; Vildagliptin

\section{INTRODUCTION}

The worldwide prevalence of type 2 diabetes mellitus (T2DM) continues to rise dramatically, with Asian countries contributing more than half of the world's diabetic population [1, 2]. Currently, 7.2 million individuals aged between 20 and 79 years are affected by T2DM in Japan [1]. T2DM clinical practice guidelines by the American Diabetes Association [3] and International Diabetes Federation [4] suggest starting treatment with metformin unless contraindicated, followed by the addition of other oral antidiabetic drugs (OADs) if patients fail to achieve glycosylated hemoglobin (HbA1c) goal $<7.0 \%$. The Japan Diabetes Society (JDS) suggests starting pharmacotherapy with any OAD depending on the physiological status of the patient after diet and exercise failure [5]. Most of the
Japanese patients with T2DM have a tendency to a low body mass index (BMI); and as insulin secretion deficiency plays a predominant role in disease pathology [6], insulin secretagogues are the preferred first-line treatment option in Japan. Recently, Japanese patients with T2DM are being increasingly treated with dipeptidyl peptidase-4 (DPP-4) inhibitors (e.g., vildagliptin) [7], which increase insulin secretion from $\beta$-cells in a glucose-dependent manner [8]. Moreover, the progressive nature of the disease warrants treatment intensification with other antidiabetic agents having complementary mechanism of action to maintain glycemic control over long term [5].

The mechanistic synergy between vildagliptin and metformin [8, 9], and the efficacy and safety of vildagliptin added to metformin in Japanese patients with T2DM inadequately controlled with metformin monotherapy has already been demonstrated [10]. However, the benefit of switching patients, who are treated with vildagliptin and require additional treatment, to vildagliptin and metformin has not been established. So far, no DPP-4 inhibitor/metformin single-pill combination (SPC) is available in Japan. Such a SPC has the additional benefit of a reduced pill burden, and potentially better compliance [11]. Moreover, the efficacy of low-dose metformin [250 mg twice daily (bid)] has not been studied previously in a randomized trial setting in Japanese patients with T2DM. Accordingly, the current study was aimed to assess the efficacy and safety of vildagliptin/metformin SPC at doses of $50 / 250$ and $50 / 500 \mathrm{mg}$ in Japanese patients with T2DM inadequately controlled with diet, exercise and vildagliptin monotherapy. 


\section{MATERIALS AND METHODS}

\section{Study Design}

This 14-week, multicenter, double-blind, parallel-group, placebo-controlled, randomized study was conducted across 30 centers in Japan from May 2013 to February 2014. Patients with T2DM inadequately controlled (HbA1c 7.0-10.0\%) with diet, exercise and vildagliptin monotherapy were eligible for inclusion. Following the screening visit (visit 1), eligible patients on vildagliptin $50 \mathrm{mg}$ bid monotherapy for at least 10 weeks proceeded directly to randomization (baseline, visit 2). Whereas patients taking other OADs were switched to vildagliptin $50 \mathrm{mg}$ bid and were asked to complete a 12-week run-in period (visit 101) before randomization (Fig. 1).

Eligible patients were randomized (2:1) to receive either vildagliptin/metformin SPC (hereafter called the vilda/met group) or vildagliptin/placebo SPC (hereafter called the vilda/placebo group). In the vilda/met treatment group, patients were randomized (1:1) to receive either vilda/met $50 / 250$ or
50/500 mg bid (Fig. 1). In the vilda/met group, all patients started double-blind treatment with vilda/met 50/250 mg bid, and patients randomized to the subgroup vilda/met 50/500 mg bid were up-titrated after 2 weeks. Efficacy and safety were assessed at baseline and at weeks $2,6,10$, and 14 . No rescue medication was allowed, and patients with unsatisfactory therapeutic effect [fasting plasma glucose $(\mathrm{FPG}) \geq 15.0 \mathrm{mmol} / \mathrm{L}]$ were discontinued from the study.

\section{Study Population}

The study included patients with T2DM aged $\geq 20$ to $<75$ years, BMI $\geq 20$ to $\leq 35 \mathrm{~kg} / \mathrm{m}^{2}$, and who were inadequately controlled $(\mathrm{HbA} 1 \mathrm{c} \geq 7.0 \%$ to $\leq 10.0 \%)$ by diet and vildagliptin $50 \mathrm{mg}$ bid monotherapy. The key exclusion criteria were: $F P G \geq 15.0 \mathrm{mmol} / \mathrm{L}$; history of type 1 diabetes, acute metabolic conditions such as ketoacidosis, lactic acidosis; patients with congestive heart failure (New York Heart Association Class III or IV); myocardial infarction, or coronary artery bypass surgery in the past 6 months, unstable angina in the past

\begin{tabular}{|l|l|l|}
\hline Screening & Run-in period & Double-blind treatment period \\
\hline
\end{tabular}

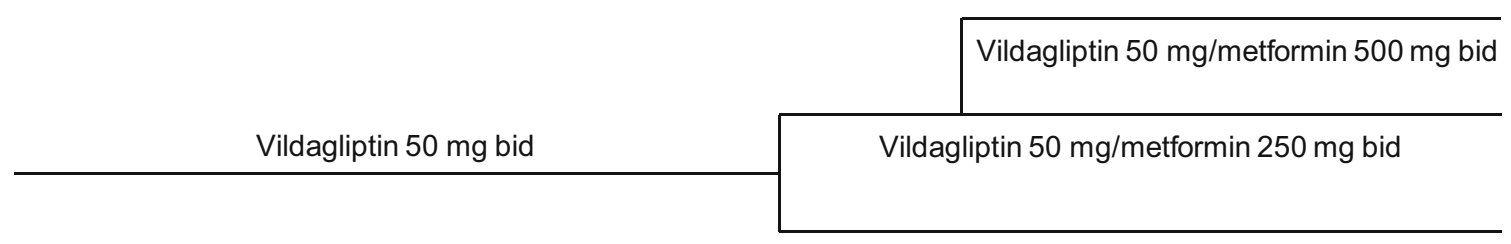

Vildagliptin $50 \mathrm{mg} /$ Placebo bid

\begin{tabular}{r|l|l|l|l|l|l|l|l|l|} 
Visit & $1^{*}$ & 101 & 102 & $103^{* \star}$ & 2 & $3^{\ddagger}$ & 4 & 5 \\
\cline { 2 - 8 } Week & -14 & -12 & -8 & -2 & $\mathrm{BL}^{+}$ & 2 & 6 & 10
\end{tabular}

\footnotetext{
*Patients who met all criteria and on stable dose of vildagliptin $50 \mathrm{mg}$ bid for at least 10 weeks proceeded directly to visit 2 (randomization). Patients at visit 1 who met all the criteria but were taking antidiabetic drugs other than vildagliptin entered the 12-week run-in period and proceeded to visit 101. ${ }^{* *}$ Eligibility assessment for patients who entered the run -in period. †Baseline, the day of randomization. ${ }^{\ddagger}$ Up titration of patients randomized to vildagliptin/metformin $50 / 500 \mathrm{mg}$ bid from vildagliptin/metformin 50/250 mg bid. Bid, twice daily
}

Fig. 1 Study design 
3 months; acute or chronic liver disease; or impaired renal function.

\section{Study Endpoints and Assessments}

Change in HbA1c from baseline to study end in all vilda/met groups was the primary efficacy endpoint. The secondary endpoints included: HbA1c change from baseline to study end in the subgroups of patients by metformin dose, percentage of patients achieving HbA1c target $(<7.0 \%) /$ reduction of $\geq 0.5 \%$ and change in FPG from baseline to study end. HbA1c values are reported in National Glycohemoglobin Standardization Program units (NGSP, \%).

Safety assessments included collecting all adverse events (AEs), serious AEs (SAEs) data with their severity and suspected relationship to the study drug, regular assessments of hematology, biochemistry, vital signs and body weight. All the laboratory assessments were performed at a central laboratory (Mitsubishi Chemical Medience Corporation, Tokyo, Japan). Patients were asked to record hypoglycemic events in a study diary. Hypoglycemia was defined as symptoms suggestive of hypoglycemia, further confirmed by self-monitored blood glucose measurement of $<3.1 \mathrm{mmol} / \mathrm{L}$. The event was considered severe if the patient required assistance of another person or hospitalization.

\section{Statistical Analysis}

Assuming a dropout rate of 5\%, 171 patients were to be randomized in a ratio of $2: 1$ (vilda/ met 114; vilda/placebo: 57) to achieve the target sample size of 162 . This sample size would ensure $90 \%$ power to detect a between-group difference of 0.6 absolute units in HbA1c at a one-sided significance level of 0.025 and a standard deviation of $1.1 \%$. The sample size of
57 patients in each vilda/met subgroup would ensure $90 \%$ power with a one-sided significance level of 0.025 to detect a reduction of 0.5 absolute units in $\mathrm{HbA1c}$ from baseline at a standard deviation of $1.0 \%$. Statistical analysis was performed using SAS 9.2 (SAS Institute Inc., Cary NC, USA).

All randomized patients who received at least one dose of the study drug and had at least one post-randomization efficacy parameter (HbA1c, FPG) assessment constituted the full analysis set (FAS). The primary and secondary efficacy analyses were based on FAS. The changes in HbA1c and FPG from baseline to study endpoint [final available assessment value at any visit up to the final visit (week 14)] reported as mean \pm SE were analyzed using the analysis of covariance model, with treatment as a classification variable and baseline value as a covariate. The last observation carried forward method was used for imputing missing data.

Chi-squared test was used to assess and compare the proportion of responders in the two groups. Safety data were summarized descriptively by treatment for the safety analysis set which included all the patients who received at least one dose of the study drug.

\section{Ethics and Good Clinical Practice}

The independent Ethics Committee/ Institutional Review Board at each center reviewed and approved the study protocol. All procedures followed were in accordance with the ethical standards of the responsible committee on human experimentation (institutional and national) and with the Helsinki Declaration of 1975, as revised in 2000 and 2008. Informed consent was obtained from all patients for being included in the study. The study is registered with ClinicalTrials.gov, identifier: NCT01811485. 


\section{RESULTS}

\section{Patient Disposition and Baseline Characteristics}

A total of 171 patients were randomized (vilda/ met, $n=115$; vilda/placebo, $n=56$ ) of which 160 (93.6\%) patients completed the study. The most common reasons for discontinuations were AEs in the vilda/met group (3.5\%) and unsatisfactory therapeutic effectiveness in the vilda/placebo group (5.4\%) (Fig. 2). Patient demographics and baseline characteristics were comparable between the two treatment groups (Table 1). The patients had an overall age (mean \pm SD) $57.0 \pm 10.5$ years, BMI $25.8 \pm 3.4 \mathrm{~kg} / \mathrm{m}^{2}$, FPG $8.8 \pm 1.8 \mathrm{mmol} / \mathrm{L}$ and T2DM duration $7.0 \pm 6.6$ years. Baseline HbA1c was similar between the two groups (Table 1). Almost $80 \%$ of the patients received concomitant medications at baseline. The most frequently used concomitant medications were lipid-lowering drugs (42.1\%) and antihypertensives (39.8\%).

\section{Efficacy}

The mean HbA1c change over 14 weeks is shown in Fig. 3a. After 2 weeks, the mean HbA1c levels were lower in all vilda/met groups compared with the vilda/placebo group. At week 14, a statistically significant between-treatment difference in (mean $\pm \mathrm{SE}$ ) HbA1c of $-1.0 \pm 0.1 \%(P<0.001)$, in favor of the vilda/met group was observed (both doses combined) (Table 2). Statistically significant

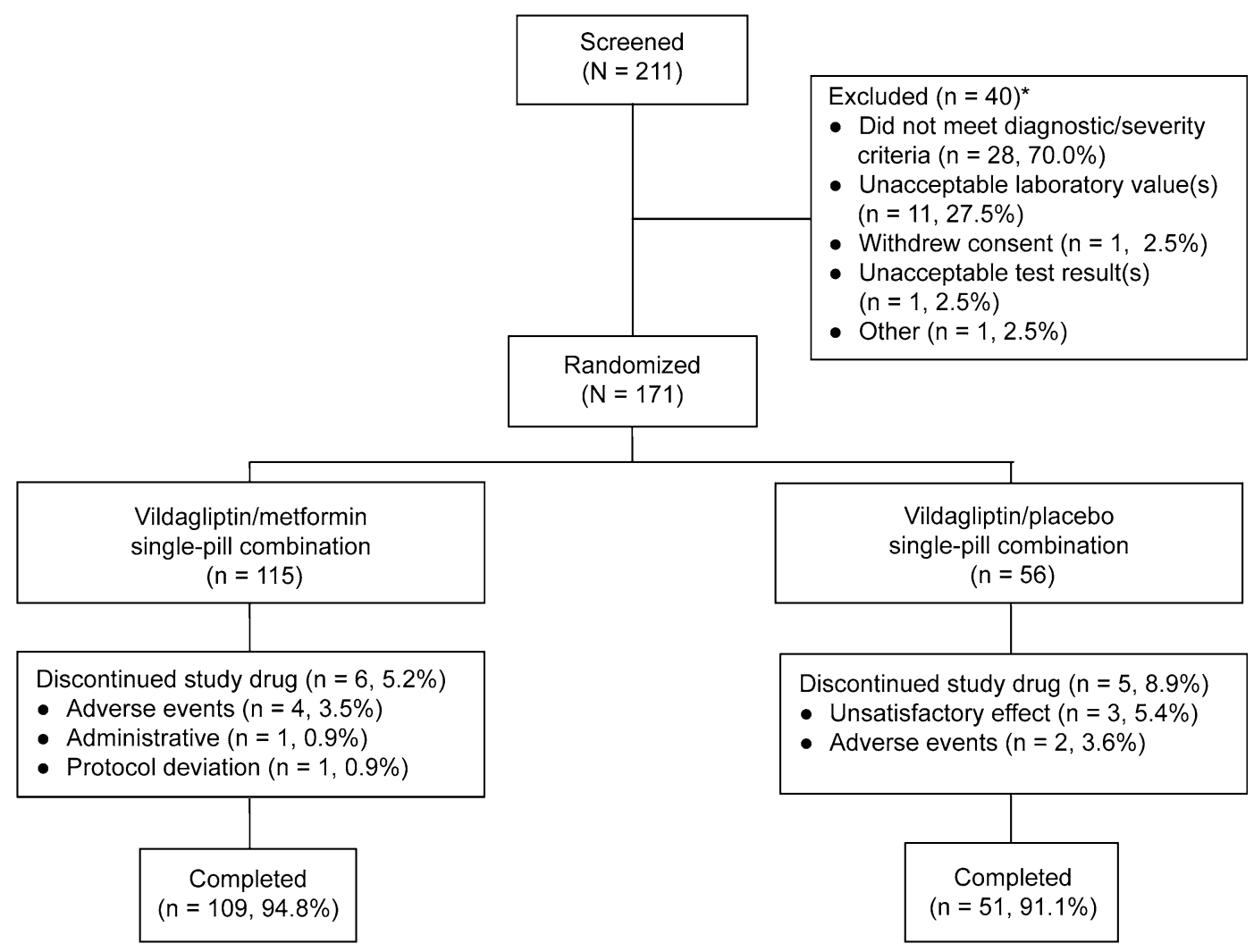

${ }^{*}$ More than one reason for discontinuation during the screening period

Fig. 2 Flow diagram depicting patient disposition 
Table 1 Patient demographics and baseline characteristics (randomized set)

\begin{tabular}{llll}
\hline Parameters & Vildagliptin/metformin & Vildagliptin/placebo & Total \\
& SPC & SPC & $\boldsymbol{N}=\mathbf{1 7 1}$ \\
& $\boldsymbol{n}=\mathbf{1 1 5}$ & $\boldsymbol{n}=\mathbf{5 6}$ & \\
\hline Age (years) & $57.5 \pm 10.9$ & $56.2 \pm 9.8$ & $57.0 \pm 10.5$ \\
$\geq 65$ years $[n(\%)]$ & $35(30.4)$ & $11(19.6)$ & $46(26.9)$ \\
Men $[n(\%)]$ & $82(71.3)$ & $40(71.4)$ & $122(71.3)$ \\
Body weight $(\mathrm{kg})$ & $69.6 \pm 12.5$ & $72.0 \pm 11.3$ & $70.4 \pm 12.1$ \\
BMI $\left(\mathrm{kg} / \mathrm{m}^{2}\right)$ & $25.5 \pm 3.4$ & $26.5 \pm 3.3$ & $25.8 \pm 3.4$ \\
HbA1c $(\%)$ & $7.9 \pm 0.8$ & $8.0 \pm 0.8$ & $7.9 \pm 0.8$ \\
$\leq 8 \%[n(\%)]$ & $77(67.0)$ & $37(66.1)$ & $114(66.7)$ \\
$>8$ to $\leq 9 \%[n(\%)]$ & $23(20.0)$ & $11(19.6)$ & $34(19.9)$ \\
$>9 \%[n(\%)]$ & $15(13.0)$ & $8(14.3)$ & $23(13.5)$ \\
FPG $(\mathrm{mmol} / \mathrm{L})$ & $8.8 \pm 1.7$ & $8.9 \pm 2.0$ & $8.8 \pm 1.8$ \\
$\geq 8.9 \mathrm{mmol} / \mathrm{L}[n(\%)]$ & $49(42.6)$ & $23(41.1)$ & $72(42.1)$ \\
Duration of T2DM (years) & $7.0 \pm 6.5$ & $7.1 \pm 6.9$ & $7.0 \pm 6.6$ \\
eGFR $(\mathrm{MDRD})\left[\mathrm{mL} / \mathrm{min} / 1.73 \mathrm{~m}^{2}, n(\%)\right]$ & & $159(93.0)$ \\
Normal $>80$ & $106(92.2)$ & $53(94.6)$ & $12(7.0)$ \\
Mild $\geq 50$ to $\leq 80$ & $9(7.8)$ & $3(5.4)$ &
\end{tabular}

Data are expressed as mean \pm standard deviation, unless specified otherwise

$B M I$ body mass index, eGFR estimated glomerular filtration rate, FPG fasting plasma glucose, $H b A 1 c$ glycosylated hemoglobin, $M D R D$ modification of diet in renal disease, SPC single-pill combination, T2DM type 2 diabetes mellitus

reductions $(P<0.001)$ in $\mathrm{HbA1c}$ from baseline were also observed for the vilda/met 50/250 mg bid and vilda/met 50/500 mg bid subgroups (Fig. 3b). The placebo-corrected difference for the change in $\mathrm{HbA} 1 \mathrm{c}$ was $-0.8 \% \quad[95 \%$ confidence interval (CI) $-1.0 \%,-0.6 \%]$ and $-1.2 \%$ (95\% CI $-1.4 \%,-1.0 \%)$ in the vilda/met 50/250 and 50/500 mg subgroups, respectively.

The proportion of patients who achieved either an HbA1c of $<7.0 \%$ or an HbA1c drop of $\geq 0.5 \%$ at week 14 was significantly higher $(P<0.001)$ for the vilda/met group compared with the vilda/placebo group (Table 3). 32.1\% and $59.3 \%$ of patients in the vilda/met $50 / 250 \mathrm{mg}$ and vilda/met 50/500 mg subgroups, respectively, achieved HbA1c $<7.0 \%$. Absolute mean changes in HbA1c from baseline to endpoint were greater in the vilda/met group for the various subgroups of patients defined by age, gender, baseline BMI, baseline HbA1c and baseline FPG. In the vilda/met group, mean reductions in HbA1c were numerically higher for patients with higher baseline HbA1c values. 

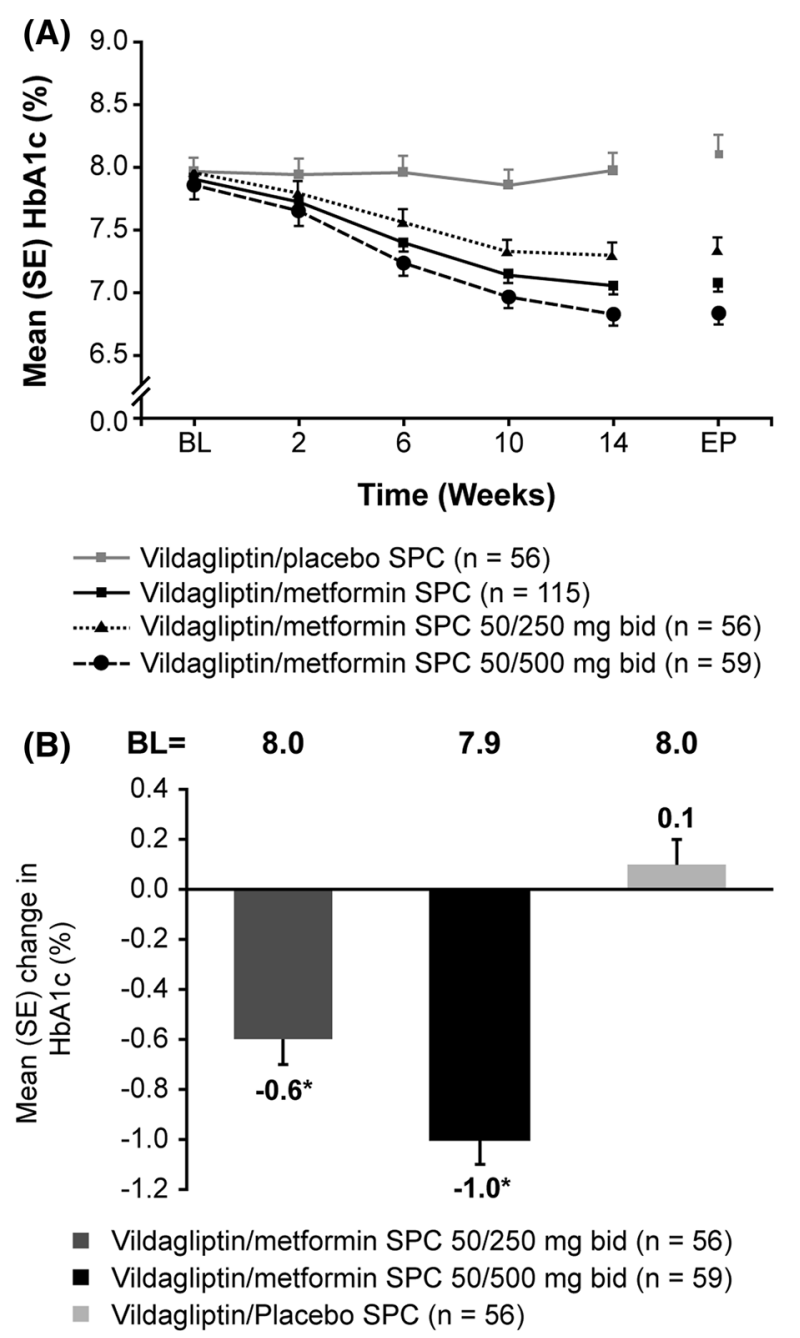

Fig. 3 a Mean glycosylated hemoglobin (HbAlc \%) by treatment and visit (full analysis set). b Adjusted mean change in HbAlc from baseline to endpoint (full analysis set). ${ }^{*} P<0.001$. $B L$ baseline, $E P$ endpoint, $S E$ standard error, $S P C$ single-pill combination

The mean FPG over 14 weeks is shown in Fig. 4. The mean change in FPG from baseline to endpoint was greater for patients receiving vilda/met $(-0.7 \pm 0.2 \mathrm{mmol} / \mathrm{L})$ compared with those receiving vilda/placebo $(0.9 \pm 0.2 \mathrm{mmol} /$ L), with a statistically significant betweentreatment difference of $-1.6 \pm 0.3 \mathrm{mmol} / \mathrm{L}$ $(P<0.001)$. The placebo-corrected reductions in FPG from baseline to endpoint were $-1.5 \pm 0.3(95 \% \mathrm{CI}-2.1,-0.8)$ and $-1.8 \pm 0.3$
(95\% CI $-2.4,-1.2) \mathrm{mmol} / \mathrm{L}$ in the vilda/met $50 / 250 \mathrm{mg}$ and 50/500 $\mathrm{mg}$ subgroups, respectively.

\section{Safety}

The overall safety profile is summarized in Table 4. The incidence of AEs was lower in the vilda/met group $(43.5 \%)$ compared with the vilda/placebo group (67.9\%). The incidences of AEs were similar between the two vilda/met subgroups $(44.6 \%$ and $42.4 \%$ for vilda/met $50 / 250$ and 50/500, respectively). Most of the AEs were mild or moderate in severity. The most frequently reported AEs by system organ class (SOC) were 'infections and infestations' (16.5\% vs. $25.0 \%)$ and gastrointestinal disorders (16.5\% vs. $14.3 \%$ ) in the vilda/met and vilda/placebo groups, respectively. Nasopharyngitis was the most frequently reported $\mathrm{AE}$ in both the groups $(9.6 \%$ for vilda/met vs. $17.9 \%$ for vilda/placebo). Discontinuations due to AEs were low in both the groups $(3.5 \%$ and $3.6 \%$ in the vilda/met and vilda/placebo groups, respectively).

Three patients reported SAEs: syncope and convulsion in one patient in the vilda/met 50/500 mg subgroup; epiglottitis and gastric cancer in one patient each in the vilda/placebo group. There were no deaths in the study. There were no hypoglycemic events reported in either group. Asymptomatic mild elevations in pancreatic enzymes were reported in six patients. However, none of the events were considered as AEs of acute pancreatitis by the investigator and all patients completed the study. Body weight remained constant in both the groups after 14 weeks of treatment: $+0.1 \pm 0.1 \mathrm{~kg}$ (baseline, $69.5 \pm 12.6 \mathrm{~kg}$ ) in the vilda/met group and $+0.2 \pm 0.2 \mathrm{~kg}$ $(72.1 \pm 11.3 \mathrm{~kg})$ in the vilda/placebo group. 
Table 2 ANCOVA results for change in HbAlc (\%) from baseline to endpoint (full analysis set)

\begin{tabular}{|c|c|c|c|c|c|c|}
\hline \multirow[t]{2}{*}{ Treatment } & \multirow[t]{2}{*}{$N$} & \multirow[t]{2}{*}{$\begin{array}{l}\text { Baseline mean } \\
(\mathrm{SE})\end{array}$} & \multirow[t]{2}{*}{$\begin{array}{l}\text { Adjusted mean } \\
\text { change (SE) }\end{array}$} & \multicolumn{3}{|c|}{$\begin{array}{l}\text { Difference in adjusted mean } \\
\text { change }\end{array}$} \\
\hline & & & & $\begin{array}{l}\text { Mean } \\
\text { (SE) }\end{array}$ & 95\% CI & $P$ value \\
\hline $\begin{array}{l}\text { Vildagliptin/metformin SPC (both } \\
\text { doses combined) }\end{array}$ & 115 & $7.9(0.1)$ & $-0.8(0.1)$ & $-1.0(0.1)$ & $-1.2,-0.8$ & $<0.001$ \\
\hline Vildagliptin/placebo SPC & 56 & $8.0(0.1)$ & $0.1(0.1)$ & & & \\
\hline
\end{tabular}

ANCOVA analysis of covariance, $C I$ confidence interval, HbAlc glycosylated hemoglobin, $S E$ standard error, $S P C$ single-pill combination

Table $3 \mathrm{HbA1c}(\%)$ responder rates (full analysis set)

\begin{tabular}{lll}
\hline Responder criteria & Vildagliptin/metformin & Vildagliptin/placebo \\
& SPC & SPC \\
& $\boldsymbol{n}=\mathbf{1 1 5}$ & $\boldsymbol{n}=\mathbf{5 6}$ \\
\hline $\mathrm{HbAlc}<7.0 \%\left[n / N^{* *}(\%)\right]$ & $49 / 107(45.8)^{*}$ & $7 / 52(13.5)$ \\
Reduction of $\mathrm{HbAlc} \geq 0.5 \%\left[n / N^{\dagger}(\%)\right]$ & $85 / 115(73.9)^{*}$ & $9 / 56(16.1)$ \\
\hline
\end{tabular}

HbAlc glycosylated hemoglobin, SPC single-pill combination

${ }^{*} P<0.001$

** Denominator includes patients with baseline HbAlc $\geq 7.0 \%$ and endpoint HbAlc measurement

$\dagger$ Denominator includes patients with both baseline and endpoint HbAlc measurements

\section{DISCUSSION}

This is the first randomized clinical study of an SPC of DPP-4 inhibitor and metformin in Japanese patients with T2DM. The goal of the study was to assess the efficacy and safety of vildagliptin/metformin SPC over 14 weeks in Japanese patients with T2DM inadequately controlled by diet, exercise, and vildagliptin monotherapy.

The present study showed that vildagliptin/ metformin SPC is efficacious, safe and welltolerated in Japanese patients with T2DM. The HbA1c reduction observed with the SPC $(-0.8 \%$; baseline: $7.9 \%)$ in patients inadequately controlled by vildagliptin monotherapy was similar to the earlier reported drop in HbA1c with free-dose combination of vildagliptin/metformin in patients inadequately controlled by metformin monotherapy [10]. Almost half of patients treated with vilda/met SPC achieved the JDS recommended glycemic target of $\mathrm{HbA} 1 \mathrm{c}<7.0 \%$ [5] with three-fourths of patients demonstrating a clinically relevant drop in HbA1c $(\geq 0.5 \%)$ [12], thus, highlighting the benefit of switching patients who are inadequately controlled with vildagliptin monotherapy to the vildagliptin/ metformin SPC. The mean reduction in FPG levels was also significantly higher for the vilda/ met group compared with the vilda/placebo group, which is consistent with the mechanism of action of metformin to decrease the overnight hepatic glucose production [13]. The data presented here are the first data to establish the clinical efficacy of metformin $250 \mathrm{mg}$ bid in Japanese patients, as such closing an important gap. Even this low metformin dose resulted in 
clinically relevant glycemic benefit with an HbA1c difference of $0.8 \%$ vs. vilda/placebo group.

The overall incidence of AEs was lower in the vilda/met group compared with the vilda/placebo group. This can be mostly attributed to a higher incidence of mild events of nasopharyngitis, all of which were considered unrelated to the study drug. This is

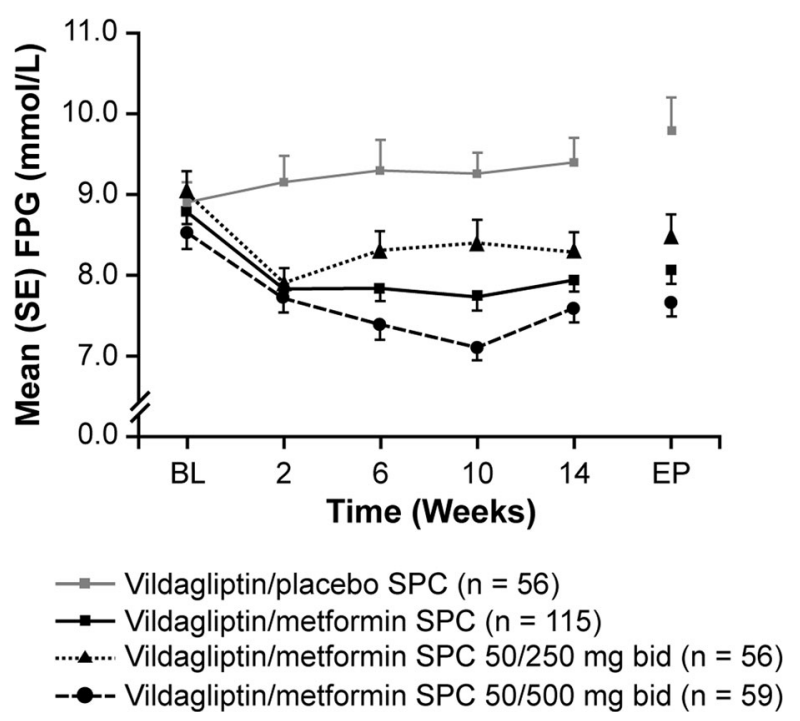

Fig. 4 Mean fasting plasma glucose $(\mathrm{mmol} / \mathrm{L})$ by treatment and visit (full analysis set). $B L$ baseline, $E P$ endpoint, SPC single-pill combination likely a chance finding, given that the only treatment change in this patient was adding placebo treatment to already existing vildagliptin treatment. There were no hypoglycemic events reported in this study, despite the significant improvement in the glycemic control with the SPC, which is consistent with the earlier known safety profile of vildagliptin in Japanese patients with T2DM [14, 15] and a potential vildagliptin mediated protective effect against hypoglycemia through enhanced gastric inhibitory polypeptide [9]. There was no weight gain over 14 weeks of treatment in both the groups reconfirming the previously established weight neutrality effect of metformin [16]. Overall, the safety and tolerability of the vilda/met group were in line with the known safety profile of vildagliptin as a single agent or as a free combination with metformin [10, 14, 15].

Treatment with SPC of vildagliptin/ metformin targets the multiple pathophysiological abnormalities of T2DM such as impaired insulin secretion, increased endogenous glucose production, and decreased utilization of glucose, in turn helping patients

Table 4 Overall summary of adverse events (safety set)

\begin{tabular}{lcllc}
\hline $\boldsymbol{n}$ (\%) & $\begin{array}{l}\text { Vildagliptin/ } \\
\text { metformin SPC } \\
(\boldsymbol{n}=\mathbf{1 1 5})\end{array}$ & $\begin{array}{l}\text { Vildagliptin/ } \\
\text { metformin SPC } \\
\mathbf{5 0 / 2 5 0 ~} \mathbf{~ g} \\
(\boldsymbol{n}=\mathbf{5 6})\end{array}$ & $\begin{array}{l}\text { Vildagliptin/ } \\
\text { metformin SPC } \\
\mathbf{5 0 / 5 0 0 ~} \mathbf{~ m g} \\
(\boldsymbol{n}=\mathbf{5 9})\end{array}$ & $\begin{array}{l}\text { Vildagliptin/ } \\
\text { placebo SPC } \\
(\boldsymbol{n}=\mathbf{5 6})\end{array}$ \\
\hline Adverse events (AEs) & $50(43.5)$ & $25(44.6)$ & $25(42.4)$ & $38(67.9)$ \\
AEs related to the study drug & $20(17.4)$ & $10(17.9)$ & $10(16.9)$ & $12(21.4)$ \\
Serious AEs & $1(0.9)$ & $0(0.0)$ & $1(1.7)$ & $2(3.6)$ \\
Discontinuation due to AEs & $4(3.5)$ & $1(1.8)$ & $3(5.1)$ & $2(3.6)$ \\
Hypoglycemia & $0(0.0)$ & $0(0.0)$ & $0(0.0)$ & $0(0.0)$ \\
Deaths & $0(0.0)$ & $0(0.0)$ & $0(0.0)$ & $0(0.0)$ \\
\hline
\end{tabular}

$A E s$ adverse events, $S P C$ single-pill combination 
to maintain good glycemic control. Metformin complements the mechanism of action of vildagliptin by raising absolute levels of glucagon like peptide-1 (GLP-1) [9]. Furthermore, SPC formulations have advantages such as reduced pill burden, improved convenience and adherence over free-dose combinations [17]. Results from a meta-analysis showed that SPC reduces the risk of non-compliance by $26 \%$ compared with the free-dose combination [11].

\section{CONCLUSION}

In conclusion, robust glucose-lowering efficacy along with good safety and tolerability makes the vildagliptin/metformin SPC an attractive treatment option for Japanese patients with T2DM who require additional treatment beyond vildagliptin monotherapy.

\section{ACKNOWLEDGMENTS}

The authors would like to thank the patients and staff who participated in this study. Sponsorship and article processing charges for this study were funded by Novartis Pharma K.K., Tokyo, Japan. All the authors meet the ICMJE criteria for authorship, participated at all stages of manuscript development and approved the final manuscript for publication. All authors had full access to all of the data and take complete responsibility for the integrity of the data and accuracy of the data analysis. The authors thank Amit Garg (Novartis Healthcare Private Limited, Hyderabad, India) for medical writing assistance.

Conflict of interest. Masato Odawara is the independent medical advisor for this study and has received consultancy fees from Novartis
Pharma K.K., Tokyo, Japan. Mika Yoshiki is an employee of Novartis Pharma K.K., Tokyo, Japan. Misako Sano is an employee of Novartis Pharma K.K., Tokyo, Japan. Izumi Hamada is an employee of Novartis Pharma K.K., Tokyo, Japan. Valentina Lukashevich is employed by and owns shares in Novartis. Wolfgang Kothny is employed by and owns shares in Novartis.

Compliance with ethics. The independent Ethics Committee/Institutional Review Board at each center reviewed and approved the study protocol. All procedures followed were in accordance with the ethical standards of the responsible committee on human experimentation (institutional and national) and with the Helsinki Declaration of 1975, as revised in 2000 and 2008. Informed consent was obtained from all patients for being included in the study. The study is registered with ClinicalTrials.gov, identifier: NCT01811485.

Open Access. This article is distributed under the terms of the Creative Commons Attribution Noncommercial License which permits any noncommercial use, distribution, and reproduction in any medium, provided the original author(s) and the source are credited.

Open Access. This article is distributed under the terms of the Creative Commons Attribution Noncommercial License which permits any noncommercial use, distribution, and reproduction in any medium, provided the original author(s) and the source are credited.

\section{REFERENCES}

1. International Diabetes Federation. IDF Diabetes Atlas, 6th ed. Brussels, Belgium: International Diabetes Federation; 2013. http://www.idf.org/ diabetesatlas/download-book. Accessed 4 Feb 2014. 
2. Wild S, Roglic G, Green A, Sicree R, King H. Global prevalence of diabetes: estimates for the year 2000 and projections for 2030. Diabetes Care. 2004;27:1047-53.

3. American Diabetes Association. Standards of medical care in diabetes-2014. Diabetes Care. 2014;37:S14-80.

4. International Diabetes Federation. Global guideline for type 2 diabetes. Diabetes Res Clin Pract. 2014;104:1-52.

5. Japan Diabetes Society, Treatment Guide for Diabetes edited by Japan Diabetes Society 2012-2013. Bunkodo Co. Ltd.; 2013 http://www. jds.or.jp/modules/en/index.php?contentid=27. Accessed 4 Feb 2014.

6. Fukushima $M$, Usami $M$, Ikeda $M$, et al. Insulin secretion and insulin sensitivity at different stages of glucose tolerance: a cross-sectional study of Japanese type 2 diabetes. Metabolism. 2004;53:831-5.

7. Oishi M, Yamazaki K, Okaguchi F, Sugimoto H, Kanatsuka A, Kashiwagi A, Japan Diabetes Clinical Data Management Study Group. Changes in oral antidiabetic prescriptions and improved glycemic control during the years 2002-2011 in Japan (JDDM32). J Diabetes Investig. 2014;5:581-7.

8. Ahrén B, Schweizer A, Dejager S, Villhauer EB, Dunning BE, Foley JE. Mechanisms of action of the dipeptidyl peptidase-4 inhibitor vildagliptin in humans. Diabetes Obes Metab. 2011;13:775-83.

9. Ahrén B, Foley JE, Bosi E. Clinical evidence and mechanistic basis for vildagliptin's action when added to metformin. Diabetes Obes Metab. 2011;13:193-203.
10. Odawara M, Hamada I, Suzuki M. Efficacy and safety of vildagliptin as add-on to metformin in Japanese patients with type 2 diabetes mellitus. Diabetes Ther. 2014;5:169-81.

11. Bangalore S, Kamalakkannan G, Parker S, et al. Fixed-dose combinations improve medication compliance: a meta-analysis. Am J Med. 2007;120:713-9.

12. Little RR, Rohlfing CL, Sacks DB, et al. Status of hemoglobin A1c measurement and goals for improvement: from chaos to order for improving diabetes care. Clin Chem. 2011;57:205-14.

13. Kirpichnikov D, McFarlane SI, Sowers JR. Metformin: an update. Ann Intern Med. 2002;137:25-33.

14. Kikuchi M, Abe N, Kato M, Terao S, Mimori N, Tachibana H. Vildagliptin dose-dependently improves glycemic control in Japanese patients with type 2 diabetes mellitus. Diabetes Res Clin Pract. 2009;83:233-40.

15. Odawara M, Suzuki M, Hamada I, Iguchi A. Clinical evaluations of the vildagliptin combination therapy in type 2 diabetes patients-a long term safety study of 52 weeks treatment with vildagliptin as add-on therapy with metformin, TZD, a-GI or Glinides. J New Rem Clin. 2012;12:2593-611 (article in Japanese).

16. Golay A. Metformin and body weight. Int J Obes (Lond). 2008;32:61-72.

17. Hutchins V, Zhang B, Fleurence RL, Krishnarajah G, Graham J. A systematic review of adherence, treatment satisfaction and costs, in fixed-dose combination regimens in type 2 diabetes. Curr Med Res Opin. 2011;27:1157-68. 